\title{
ALIGNMENT OF DUST GRAINS BY MAGNETIC RELAXATION: A COMPARISON BETWEEN RESULTS OBTAINED BY TWO DIFFERENT METHODS
}

\author{
P. CUGNON
}

Observatoire Royal de Belgique, Belgium

\begin{abstract}
This paper is devoted to a comparison between results obtained by Purcell and Spitzer (1971) using a Monte-Carlo method and by the author (1971) using a Fokker-Planck equation. It is shown that there is a good agreement between the results within the dispersion expected from the Monte-Carlo method.
\end{abstract}

In a preceding paper (Cugnon, 1971) I tried to make a comparison between the results obtained by Purcell (1969) using a Monte-Carlo method for solving the problem of grain alignment by magnetic relaxation, and my own results, derived from the solution of a Fokker-Planck equation. For reasons explained in this paper, the comparison, limited to a few cases, could not be considered significant. At the same time, Purcell and Spitzer (1971) published a more detailed paper on the subject, using the same method, but with somewhat different working hypotheses. The following comparison between the Monte-Carlo and the Fokker-Planck methods will refer only to the Purcell and Spitzer paper of 1971 and the author's paper of the same year.

Table I summarizes the main hypotheses made in both methods and the essential limitations in the theories. These arise either from approximations (solution of the Fokker-Planck equation in linear cases - 'sites' approximation in the collision model used in the Monte-Carlo method) or from a lack of precision (increasing dispersion in the results given by the Monte-Carlo method, for decreasing values of $\delta$ ) (Spitzer, 1972). The definition of $\delta$ and some other quantities is shown in Table II. From Table I two important differences appear between the characteristics of both methods:

(1) The use of different shapes.

(2) The limitations of the theories, which indicate that the Monte-Carlo and the Fokker-Planck methods are complementary rather than overlapping. Our aim is to

TABLE I

\begin{tabular}{|c|c|c|}
\hline & $\begin{array}{l}\text { Monte-Carlo method } \\
\text { (Purcell and Spitzer) }\end{array}$ & $\begin{array}{l}\text { Fokker-Planck method } \\
\text { (Cugnon) }\end{array}$ \\
\hline Grain shape & square prisms & spheroids \\
\hline Collision type & 'sticking' and evaporation & $\begin{array}{l}\text { 'sticking' and evaporation or } \\
\text { specular reflection }\end{array}$ \\
\hline $\begin{array}{l}\text { Domains of } \\
\text { best accuracy }\end{array}$ & $\begin{array}{l}\text { moderate and high } \\
\text { elongation or flatness and } \\
\delta>1 .\end{array}$ & $\begin{array}{l}\text { nearly spherical grains } \\
\text { any value of } \delta \text {, or } \\
\delta \ll 1 \text {, any value of the eccentricity. }\end{array}$ \\
\hline
\end{tabular}


TABLE II

Definition of some parameters

$$
\begin{aligned}
\delta & =\frac{\text { kinetic relaxation time }}{\text { magnetic relaxation time }} \\
\xi & =\frac{2 T_{\mathrm{gra}}}{T_{\mathrm{gra}}+T_{\mathrm{gas}}} \\
T_{\mathrm{gra}} & =\text { internal temperature of grains } \\
T_{\mathrm{gas}} & =\text { kinetic temperature of gas } \\
Q_{A} & =<\frac{1}{2}\left[3 \cos ^{2}(\mathrm{~B}, \text { axis of the grain })-1\right]>
\end{aligned}
$$

study here the influence of these differences on the results, and, from this discussion, to decide if a comparison remains possible.

Table III shows a comparison between the main shape parameters of spheroids and square prisms; the first column give the aspect ratio which is, for spheroids, the ratio between the transverse axis and the axis of symmetry, and, for prisms, the ratio between the side of the square and the height of the prism. Using this definition, the inertial ratio $\gamma$ has the same value for both shapes. The third and fourth columns give, respectively for spheroids and prisms, the product $\gamma \varepsilon$, which may be defined as the ratio of the relative increments to the axial and the transverse components of the angular momentum (for a more explicit definition, see Cugnon, 1971). This parameter appears explicitly in the Fokker-Planck equation; it may be considered as a 'hidden' parameter in the Monte-Carlo approach. There is a difference between the values of this parameter, but, fortunately, the effect of this difference may be easily studied by the Fokker-Planck method in the case of infinite needles, i.e. where this difference is the highest, because for this case an analytical solution exists. After some calculation it appears that this effect is negligible. Somewhat arbitrarily, but with a very small risk of error, we assume that this holds true also for finite elongations. It then seems permissible to compare the respective results of spheroids and prisms.

\begin{tabular}{|c|c|c|c|c|}
\hline & \multirow[t]{2}{*}{$a / b$} & \multirow[t]{2}{*}{$\gamma$} & \multicolumn{2}{|l|}{$\gamma \varepsilon$} \\
\hline & & & spheroids & prisms \\
\hline \multirow[t]{3}{*}{ needles } & 0 & $\infty$ & 1 & 1.667 \\
\hline & 0.2 & 13 & 1.012 & 1.432 \\
\hline & 0.5 & 2.5 & 1.043 & 1.196 \\
\hline \multicolumn{5}{|l|}{ spheres } \\
\hline \multirow[t]{2}{*}{ or cubes } & 1 & 1 & 1 & 1 \\
\hline & 4 & 0.531 & 0.616 & 0.739 \\
\hline \multicolumn{5}{|c|}{ disks or } \\
\hline flat prisms & $\infty$ & 0.5 & 0.5 & 0.5 \\
\hline
\end{tabular}

The second difficulty arises from the different domains of values of the parameter $\delta$ covered by both methods. This difficulty can be overcome by considering the Fokker-

TABLE III

Shape parameters of spheroids and square prisms 
Planck results as a limiting case, and by checking if there is a good convergence of the Monte-Carlo results to the thus defined limit.

In practice, this may be done by looking at the values of $Q_{A} / \delta$ given by the MonteCarlo method, $Q_{A}$ being the degree of alignment of the grains, and by studying the convergence of a sequence of those values for a given aspect ratio, to the parameter

$$
\lim _{\delta \rightarrow 0} \frac{Q_{\mathrm{A}}}{\delta}=\frac{3}{2} F(1-\xi)
$$

which is given by the Fokker-Planck equation method. The results shown in Figure 1 demonstrate that the convergence is quite good within the dispersion in the MonteCarlo results, and, furthermore, confirms the author's prediction (1971) i.e. that $Q_{A}$ remains proportional to $\delta$ for $\delta \lesssim 1$, for moderate eccentricities.

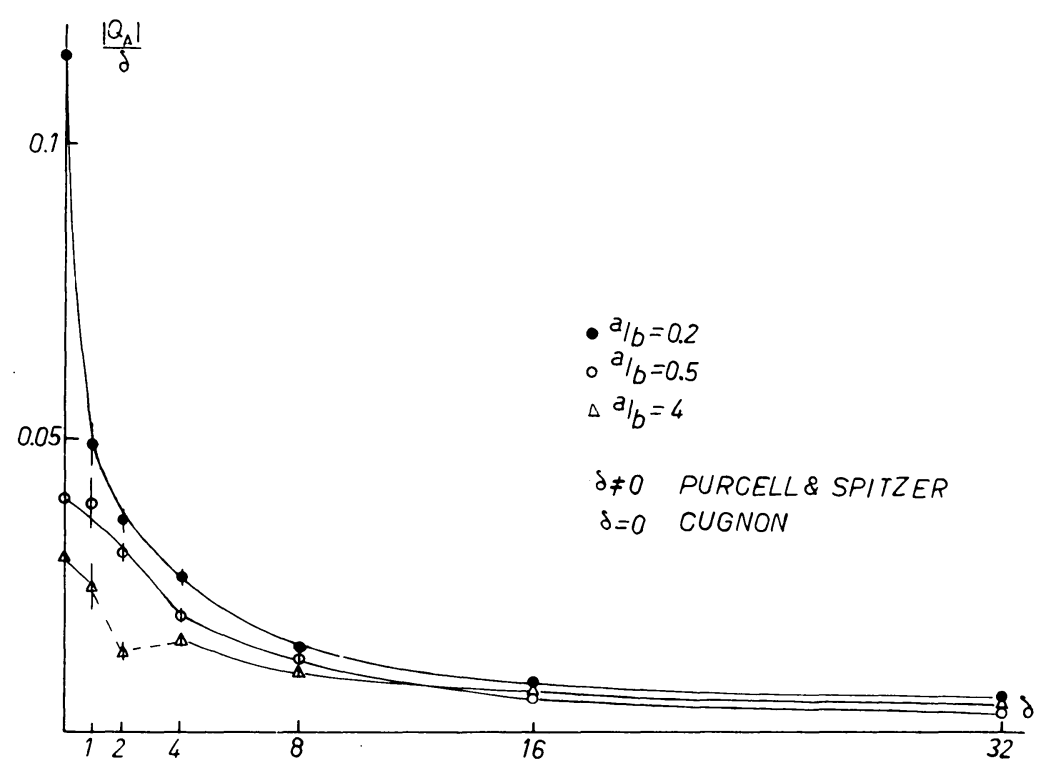

Fig 1

\section{Acknowledgement}

I am much indebted to Prof. L. Spitzer Jr., who suggested this work, and gave me many helpful remarks and suggestions.

\section{References}

Cugnon, P.: 1971, Astron. Astrophys. 12, 398.

Purcell, E. M.: 1969, Physica 41, 100.

Purcell, E. M. and Spitzer, L., Jr.: 1971, Astrophys. J. 167, 31. 